\title{
IS THE DYNAMICAL HISTORY AT ODDS WITH THE
}

\section{CHEMICAL HISTORY?}

\section{F. MATTEUCCI}

Dipartimento di Astronomia, Universita' di Trieste

Via G.B. Tiepolo, 11 TRIESTE

\section{Formation and Evolution of the Galaxy as suggested by the correlation between abundances and kinematics}

The paper of Eggen, Lynden-Bell and Sandage (1962)(hereafter ELS) titled "Evidence from the motion of old stars that the Galaxy collapsed" was the first attempt to understand the formation and evolution of our Galaxy. From a study of a kinematically selected sample of high velocity stars, ELS had found a remarkable correlation between chemical abundance and orbital eccentricity, in the sense that stars with the largest ultraviolet excess (a measure of stellar metallicity, in particular Fe), i.e. the lowest metallicity, are invariably moving in highly elliptical orbits. As the average $\langle[\mathrm{Fe} / \mathrm{H}]\rangle$ (in the usual notation $[\mathrm{Fe} / \mathrm{H}]=\log (\mathrm{Fe} / \mathrm{H})_{*}-\log (\mathrm{Fe} / \mathrm{H})_{\odot}$ )is expected to increase with time, as a consequence of the progressive chemical enrichment of the gas, stars with the lowest $[\mathrm{Fe} / \mathrm{H}]$ are, on average, the oldest. ELS also found a correlation between abundance and motion of stars perpendicular to the Galactic plane. This correlation suggests a continuous decrease of the perpendicular velocity with decreasing $[\mathrm{Fe} / \mathrm{H}]$. To explain these relations ELS proposed that the Galaxy collapsed from a protocloud to a thin disk on a timescale of a few times $10^{8}$ years, with progressive chemical enrichment as the collapse proceeded. This model was subsequently criticized mainly because of selection effects in their data, i.e. given the data available to ELS one would not expect the sample to contain low abundance, low orbital eccentricity objects even if they existed. They would be absent from the high velocity catalog they used. In addition, the ELS simple model did not account for the fact that almost half of the halo stars have retrograde orbits . This fact led Larson (1969) to consider models of clumpy and turbulent protogalaxies with collapse times that sometimes exceeded $1 \mathrm{Gyr}$. 
Searle and Zinn (1978) expressed a different point of view relative to ELS as a result of their studies of the globular cluster system of the Galaxy. First they found no gradient in metallicity in the globular clusters at galactocentric distances greater than $10 \mathrm{Kpc}$. However this observation per se does not argue against the rapid collapse proposed by ELS, since a metallicity gradient is favored by a slow dissipative collapse. The second point was the problem of the Horizontal Branch morphology in globular clusters, i.e. the fact that it appears to be a function of galactocentric distance. This was attributed to age differences implying that globular clusters in the outer halo have an age spread of a couple of billion years, whereas those of the inner halo have a spread in age of less than $1 \mathrm{Gyr}$. They also claimed that for halo abundances $([\mathrm{Fe} / \mathrm{H}]<-1.0)$ there is no dependence of the kinematics on abundance for subdwarf samples when all the velocity components are considered. Therefore, they proposed a major modification of the ELS picture in which as well as a central collapse they envisaged gaseous fragments in the outer halo which remained distinct and experienced their own star formation and chemical evolution over a relatively long period before falling into equilibrium with the inner Galactic regions. In this way, one should expect no gradient and no correlation between kinematics and abundance as well as an age spread for the globular cluster population of the outer halo.

Yoshii and Saio (1979), analysing a large sample of stars, concluded that the kinematics can be better explained by a slow collapse model, with the collapse lasting $3 \cdot 10^{9}$ years.

Later, Gilmore and Reid (1983) and Gilmore and Wyse (1985) have suggested the existence of a possible third major component of the Galaxy. This third component was called thick disk. This component, which possesses some properties of the disk subsystem of globular clusters discussed by Zinn (1985), was excluded by ELS since they used catalogs containing only samples of the kinematic extremes.

Sandage (1987) and Sandage and Fouts (1987) contested the view of Searle and Zinn (1978). They presented new kinematically selected data obtained to increase the ELS sample. The new feature, not contained in ELS, was the large population of intermediate velocity stars. This component has a metallicity distribution intermediate between that of the old thin disk (e-folding scale height of $\simeq 270 p c$ ) and the extended halo (scale height $\simeq 3.2 \mathrm{Kpc}$ ) and an e-folding scale-height of $920 \mathrm{pc}$. The mean metallicity of the thick disk is $[\mathrm{Fe} / \mathrm{H}]=-0.6 \mathrm{dex}$, whereas that of the halo is $-1.5 \mathrm{dex}$ and that of the thin disk -0.1 dex. They identified this component as the thick disk of Gilmore, Reid and Wyse. The main conclusion of Sandage was that the addition of the thick disk to the thin disk and halo changes the details of ELS picture but not its essential substance. The conclusions 
of Sandage were again in contrast with those of Searle and Zinn and Norris (1986) who claimed no correlation between metallicity and kinematics below $[\mathrm{Fe} / \mathrm{H}]<-1.0$, while he instead found a continuous correlation. If Sandage is correct the chemical evolution of our Galaxy can be thought of as caused by separate processes or as part of a continuum which has different enrichment rates per unit gas mass relative to the collapse rate as a function of time. In the beginning the collapse must have been rapid with a very small energy dissipation rate, then the dissipation increased thus decreasing the collapse rate. The metal enrichment went up and so the ratio between the collapse and enrichment rate flattened thus giving higher metallicity per unit mass in stars in the flattened structures (thick disk and thin disk). Each component will therefore have a different average metallicity, chemical gradient and metallicity distribution.

On the other hand, in agreement with the previous study of Norris (1986), Ryan and Norris (1991) concluded from a kinematical study of metal poor stars that their data are not consistent with a model in which the halo formed from star formation in a dissipating collapsing cloud, unless the kinematics have since been modified by unstable orbits or violent relaxation. They suggest a picture in which numerous independently evolving clouds formed the halo stars, similar to Larson's (1969) early suggestion.

Therefore, a word of caution is in order concerning the interpretation of these kinematical data in terms of Galaxy formation mechanisms and timescales. As Gilmore et al. (1989) have pointed out, the presence or absence of a correlation between rotation velocity and stellar metallicity does not really allow one to distinguish between fast and slow collapse models of the Galaxy. So while we may learn something about the importance of energy dissipation and the efficiency of violent relaxation, the timescales enter the interpretation of the relation between rotational velocity and metallicity only by assumption.

Before trying to reach a conclusion about the halo formation we should, however, discuss another independent clock for establishing the timescale of the halo collapse, and this is given by purely chemical arguments, as will be seen in the next paragraph.

\section{Formation and Evolution of the Galaxy as suggested by abun- dance ratios}

An independent way of deducing the timescale for the halo collapse is to look at the evolution of abundance ratios determined for stars in the solar vicinity. In recent years, a great deal of observational work has been devoted to the study of the chemical abundances in solar neighbourhood stars. In particular, in the last ten years the development of efficient spectrom- 
eters combined with powerful linear detectors have revolutionized stellar spectroscopy. At the same time, theoretical model atmosphere and synthetic spectrum calculations have been considerably improved, especially with improved treatment of the line blanketing by atoms and molecules. The claimed error in the derived abundances have decreased from roughly $\simeq 0.3$ dex (a factor of 2) down to $\simeq 0.05$ dex in the most recent studies (Edvardsson et al., 1993).

A particularly useful abundance ratio is that between $\alpha$-elements and Fe since the $\alpha$-elements (i.e. those synthesized from $\alpha$ particles such as $\mathrm{O}$, $\mathrm{Ne}, \mathrm{Mg}, \mathrm{Si}$ and $\mathrm{S}$ ) are almost entirely produced by massive short living stars $\left(M>10 M_{\odot}\right.$ and lifetimes in the range $10^{6}-10^{7}$ years) exploding as type II SNe, whereas $\mathrm{Fe}$ is produced both by type II supernovae ( $\mathrm{SNe}$ ) and by $\mathrm{SNe}$ of type Ia which are believed to originate from white dwarfs in binary systems (the lifetimes go from $10^{7}$ to $10^{10}$ years and more). The most popular models for the progenitors of type Ia SNe all involve white dwarfs in binary systems which reach the Chandrasekhar mass limit by accretion from either another white dwarf or a red giant. Because of the time delay in the bulk of production of iron relative to the $\alpha$-elements, the abundance ratio $[\alpha / \mathrm{Fe}]$ can be used as a cosmic clock. Let us take oxygen as representative of $\alpha$-elements. One can infer the timescale of the halo collapse and formation by looking at the observed $[\mathrm{O} / \mathrm{Fe}]$ vs. $[\mathrm{Fe} / \mathrm{H}]$ relation for solar neighbourhood stars. This relation shows a plateau extending roughly up to $[\mathrm{Fe} / \mathrm{H}]=-1.0$ (which is also the transition metallicity from halo to disk stars) and then the $[0 / \mathrm{Fe}]$ ratio declines until it reaches the solar value. If we have a Galactic chemical evolution model following the evolution of single chemical abundances and we are able to reproduce the observed relationship, then we know at which time the gas has reached the metallicity -1.0 and this is the timescale for the formation of the halo, under the assumption that the various Galactic components formed in a continuous process. The theoretical $[\mathrm{O} / \mathrm{Fe}]$ vs. $[\mathrm{Fe} / \mathrm{H}]$ relation depends on the assumed $\mathrm{SN}$ progenitors, stellar nucleosynthesis and star formation history. For this reason, the observed abundance patterns, when compared to theoretical models, can constrain SN progenitors, nucleosynthesis and star formation. Chemical evolution models (Matteucci and François, 1992) suggest this timescale to be not longer than 1-1.5 Gyr. In particular, Matteucci and François (1992) tried to reproduce the change in slope of $[\mathrm{O} / \mathrm{Fe}]$ vs. $[\mathrm{Fe} / \mathrm{H}]$ relation at $[\mathrm{Fe} / \mathrm{H}] \simeq-1.7$, as claimed by Bessel et al. (1991). They presented models assuming different star formation laws and concluded that the data are best reproduced by a model where the star formation rate depends almost linearly upon the surface gas density. In this case the time necessary to reach the halo-disk transition metallicity $[\mathrm{Fe} / \mathrm{H}]=-1.0$ is between 1 and 1.5 Gyrs. On the other hand, if star formation has a quadratic dependence on 
the surface gas density (Schmidt law) then this time scale is only $3 \cdot 10^{8}$ years and the change in slope in the predicted $[\mathrm{O} / \mathrm{Fe}]$ vs. $[\mathrm{Fe} / \mathrm{H}]$ relation occurs not before $[\mathrm{Fe} / \mathrm{H}]=-1.0$. Therefore, it is extremely important to have detailed abundance ratios involving oxygen and the other $\alpha$-elements especially in halo stars. This timescale of 1-1.5 Gyr is longer than that inferred by ELS but is still too low to explain the age-gap of 3-4 Gyr claimed for the globular clusters by Bolte (1987). However, Vanden Bergh et al. (1990) have shown that most of the halo population of globular clusters should be coeval with a fraction of the more metal rich clusters showing ages which are perhaps 2-3 Gyr younger. More recently, Buonanno et al. (1994) showed that four globular clusters are unequivocally younger by 3-4 Gyr than others of similar metallicity and they are all at galactocentric distances larger than $20 \mathrm{Kpc}$, and concluded that is possible that they have all been accreted by the Milky Way since they are all on similar orbits. The same age spread seems to be suggested also for halo field stars (subdwarfs) (Schuster and Nissen, 1989). These authors found an age-metallicity relation for halo stars indicating a difference of $\simeq 3 \mathrm{Gyr}$ passing from $[\mathrm{Fe} / \mathrm{H}]=-3.0$ to -2.0 . If this result is real and is not affected by systematic errors, it is favoring a coherent very slow dissipative collapse of the halo. However, a time scale of the order of 2-3 Gyr is clearly at variance with that deduced from the $[\mathrm{O} / \mathrm{Fe}]$ vs. $[\mathrm{Fe} / \mathrm{H}]$ ratio. In order to have such a slow halo collapse there should be a much more restricted region of constant $[\mathrm{O} / \mathrm{Fe}]$ for halo stars. In fact, if the halo collapse time is longer than $3 \mathrm{Gyr}$ then SNe Ia had time to restore the bulk of $\mathrm{Fe}$, since the characteristic time scale for this restoration is $\simeq 1 \mathrm{Gyr}$, and the change in the slope in the observed $[0 / \mathrm{Fe}]$ should occur at much lower metallicities than observed $([\mathrm{Fe} / \mathrm{H}]=-1.0--1.5)$, as has been shown by Matteucci and Brocato (1990). Recently Marquez and Schuster (1994) seem to find that there is not an age-metallicity relation in halo stars as found before, and that the inner part of the halo is older and with less age spread than the outer one, thus confirming the inside out scenario.

Another aspect of the $[\mathrm{O} / \mathrm{Fe}]$ vs. $[\mathrm{Fe} / \mathrm{H}]$ relation, which might be in contrast with the chaotic scenario of Searle and Zinn, is the small spread around $[\mathrm{Fe} / \mathrm{H}]=-1.0$. In fact, if the halo or part of it were the result of random evolution of independent clouds, we should perhaps expect a larger spread in the $[\mathrm{O} / \mathrm{Fe}]$ ratio in halo stars, especially because of the different metallicities at which the change in the $[0 / \mathrm{Fe}]$ ratio would occur in the different clouds (Wyse and Gilmore, 1993).

These discrepancies could be eliminated if we think not in terms of a unique timescale for the halo collapse but assume that the inner halo evolved more rapidly than the outer halo, and that the chemically derived timescale refers mostly to the inner halo, due to the fact that the halo stars 
observed in the solar region are mostly those born inside the solar circle (Grenon 1987). This agrees with the fact that the observed spread in the age of globular clusters refers only to objects belonging to the outer halo. Recently, Zinn (1993) has suggested that the inner old halo clusters could have formed during a collapse not unlike the ones proposed by ELS, Larson (1976) or Sandage (1990) and Katz (1992), whereas the younger outer halo clusters could have formed in satellite systems that were accreted by the Galaxy. In the best model of Matteucci and François (1992) collapse timescales increasing with galactocentric distance are assumed. As a consequence, they predict that both halo and disk take longer to form in the outermost regions. In particular, they estimate from the chemical argument that the extreme outer halo should have formed in 3-4 Gyr as compared to the 1-1.5 Gyr predicted for the inner halo.

At present, most of the abundance data refer to stars in the solar neighbourhood, although recently abundances in disk stars at different galactocentric distances in the Galactic plane have become available. In principle, the analysis of the $[\alpha / \mathrm{Fe}]$ vs. $[\mathrm{Fe} / \mathrm{H}]$ curves for different galactocentric distances could give us an idea about the evolutionary rate in different Galactic regions.

The recent paper by Edvardsson et al. (1993) shows evidence for the $[\alpha / \mathrm{Fe}]$ ratio in metal poor disk stars $([\mathrm{Fe} / \mathrm{H}]<-0.4)$ to be smaller in stars at larger galactocentric distances. This fact also suggests that the Galaxy must have evolved less rapidly in the outer regions than in the inner ones. In such a case, in fact, it has been predicted (Matteucci, 1992) that the $[\alpha / \mathrm{Fe}]$ ratio would start decreasing at lower metallicities than in the inner regions where instead a longer plateau extending also to disk metallicities should be expected. Another claim of the paper of Edvardsson et al. is the existence of a big spread in the age- $[\mathrm{Fe} / \mathrm{H}]$ relationship, which is larger than the spread observed in the $[\alpha / \mathrm{Fe}]$ ratios. Several explanations have been proposed to explain this spread:

a) orbital diffusion combined with abundance gradients, i.e. some of the stars we observe now in the solar vicinity region have been born at different galactocentric distances where the chemical evolution had proceeded at different rates than in the solar region. In particular, the most metal deficient stars are those with the highest probability of having been born far away from the solar region. This can be the consequence of the diffusion of their orbits which amplifies the orbital eccentricity. Orbital diffusion may be the consequence of scattering of stars by molecular clouds, by massive black holes coming out of the Galactic halo, by density wavelets and their oscillations in the Galactic disk and by infalling disrupting satellite galaxies. The evidence for orbital diffusion comes from the observed relation between age and stellar velocity dispersion (Wielen 1977; Wielen et al., 1992). 
b) Discontinuous infall of primordial material,

c) local chemical inhomogeneities,

d) overlapping of stars belonging to different populations (halo, thick and thin disk) which evolved simultaneously but at different rates.

Concerning orbital diffusion coupled with abundance gradients, François and Matteucci (1993) have suggested that most of the spread observed by Edvardsson et al. in the $[\mathrm{Fe} / \mathrm{H}]$ vs. age as well as in the $[\alpha / \mathrm{Fe}]$ vs. $[\mathrm{Fe} / \mathrm{H}]$ relations can be due to the fact that some of the stars we observe at the present time in the solar neighbourhood might have been born in different regions of the Galactic disk and perhaps even in the Bulge. They assumed that the amplitude of the stellar orbits increases with the square root of their age (Wielen and Fuchs, 1985), and that the Galaxy formed from inside out, i.e. with collapse rate and star formation rate decreasing with increasing galactocentric distance. Edvardsson et al. (1993) have criticized this explanation of their data by arguing that they do not see any trend of an increasing velocity dispersion with age in the interval 3-10 Gyr, thus concluding that the heating of the disk saturates at a dispersion of about $20 \mathrm{~km} / \mathrm{sec}$ after $3 \mathrm{Gyr}$. However, in this context one has then to find a different heating process for the thick disk stars, which are at least $10 \mathrm{Gyr}$ old with a velocity dispersion of $40 \mathrm{~km} / \mathrm{sec}$. Their main argument is, however, that the spread does not change when one considers stars restricted to the solar circle. Their argument is as follows: since the mean galactocentric radius of the orbit does not change much during the history of the disk one should expect a smaller scatter for the solar circle stars if orbital diffusion was important. On the other hand, Fuchs et al. (1994) using Edvardsson et al.'s data and other data on local stars concluded that the velocity dispersion increases continuously with the stellar age, in very good agreement with predictions from the theory of orbital diffusion. In addition, by assuming an Fe gradient in the disk of the order of $-0.1 \mathrm{dex} / \mathrm{kpc}$ they found very good agreement between the observed and predicted spread in $[\mathrm{Fe} / \mathrm{H}]$. In addition, they argued that the assumptions concerning the mean orbital galactocentric distance cannot be used to trace past positions, since, while statistically the mean orbital galactocentric distance (i.e. the average between the pericentric and apocentric distance) is equal to its present value, there is an ever increasing probability looking backwards in time of scattering events from nearby orbits into the present one.

Concerning the overlapping of stars belonging to different Galactic components, Pardi et al. (1994) calculated a model for the chemical evolution of the solar neighbourhood region where the three components, halo, thick and thin disk were considered. The three components evolve at different rates but they are connected through gas flows, in the sense that during the halo formation gas is lost to form the thick disk which then accumulates 
with a time delay relative to the halo. The thin disk forms with an even longer time delay since they assumed a progressively longer timescale for the formation of the different components. However, while the timescale for the formation of the thick disk is similar to that of the halo since the gas lost from the halo goes directly to form the thick disk, the difference between the thin disk and the halo, on the other hand, is larger since the rate of formation of the thin disk depends only on the gas lost from the thick disk. Thus the thick disk is an intermediate step which allows one to separate the halo from the thin disk evolution. As a result of this the thin disk forms out of already enriched material and on a timescale as long as 3-4 Gyr thus ensuring a fit to the G-dwarf distribution. In addition, this model predicts that the $[0 / \mathrm{Fe}]$ vs. $[\mathrm{Fe} / \mathrm{H}]$ relation is not unique but is given by the convolution of the $[0 / \mathrm{Fe}]$ vs. $[\mathrm{Fe} / \mathrm{H}]$ relations for each Galactic component. In other words, this kind of model assumes an evolution in parallel, as opposed to an evolution in series as in the model of Matteucci and François. In particular, no spread at low metallicities is predicted whereas for $[\mathrm{Fe} / \mathrm{H}]>-1.0$ a spread of the order of $0.3-0.4 \mathrm{dex}$ is found. The reason for this spread resides in the fact that the chemical evolution of the gas in the halo and in the thick disk continues until the present time although the amount of star formation becomes negligible after $2 \mathrm{Gyr}$ in the halo and after $8 \mathrm{Gyr}$ in the thick disk. Therefore, very few halo stars for $[\mathrm{Fe} / \mathrm{H}]>-1.0$ and very few thick disk stars for $[\mathrm{Fe} / \mathrm{H}]>-0.5$ are predicted, so that they cannot explain all of the observed spread but probably part of it. A weakness of this model is represented by the thick disk which forms too quickly to fit well the metallicity distribution of thick disk stars, indicating that an even larger time delay between halo and thick disk formation is required. The physical reason for such a delay is the progressive increase of energy dissipation as the collapse proceeds.

\section{Abundance gradients and the formation of the galactic disk}

The existence of abundance gradients of $\mathrm{O}, \mathrm{N}, \mathrm{Si}$ and $\mathrm{S}$ along the galactic disk is shown by abundance measurements in HII regions (see Wilson and Matteucci, 1992 for a review). The existence of an iron gradient, on the other hand, is shown by stellar photometric studies of open clusters and from spectroscopic studies of late type supergiants. These gradients show that the chemical abundances decrease towards the outermost Galactic regions. From a theoretical point of view abundance gradients can be explained under different assumptions, but one of the most realistic is that the disk formed from inside out, in the sense that the star formation rate and the collapse rate were higher in the central than in the external parts. As a consequence of this, the external regions of the Galaxy may still be 
collapsing now. Therefore, these different evolutionary rates create the differences in the abundances, due to the different age-metallicity relations in the different Galactic regions. This conclusion agrees with chemo-dynamical Galactic models (Burkert et al. 1992) and with the recent data from Edvardsson et al. (1993).

Concerning the time scale for the formation of Galactic disk in the solar vicinity, chemical models (Matteucci and Tornambè 1985; Matteucci and François 1989; Ferrini et al. 1992) suggest a value of the order of 3-4 Gyr. This value comes from fitting the G-dwarf distribution in the solar neighbourhood. In fact, the well known G-dwarf problem, i.e. the lack of metal poor disk stars in the solar neighbourhood relative to the predictions of the simple model, can be solved by assuming a slow dissipative formation of the disk. An even longer timescale (4-6 Gyr) is suggested by the chemodynamical models of Burkert et al. (1992). Unfortunately, Burkert et al. did not study the formation of the halo, but started from a hot protodisk and followed only the subsequent dissipative formation of the thick and thin disk.

\section{Chemical evolution of the Galactic bulge}

In the past, the formation and evolution of the Galactic bulge has not received much attention as the formation of the halo and the disk, perhaps because halo and bulge were considered as a single spheroidal component. Now we have evidence suggesting that the bulge might have evolved independently of the halo and this evidence is in the form of chemical and kinematical studies of the bulge stellar populations.

The Milky Way bulge is a region within $1 \mathrm{kpc}$ from the center, where a metal rich, rotationally supported stellar population resides. Rich (1988) determined $[\mathrm{Fe} / \mathrm{H}]$ for $88 \mathrm{~K}$ giants in Baade's window and showed that the central stellar bulge of the Milky Way is a distinct stellar population with an age of the order of $10 \mathrm{Gyr}$ and abundance range from -1.5 to +1.0 dex with a peak between +0.15 and +0.3 dex. This result was later confirmed by Geisler and Friel (1992). This stellar distribution is easily reproduced by models assuming a fast $(\simeq 0.5 \mathrm{Gyr})$ formation time for the bulge (Matteucci and Brocato, 1990). These same models predict that most of the stars should show enhanced $\alpha$-elements relative to iron.

This result is due to the nucleosynthesis scenario presented before, coupled to the assumption of a rapid star formation and collapse.

Alternatively, if the Edmunds et al. (1991) suggestion that the [0/Fe] trend in the solar vicinity can be due to a secondary-like behaviour of $\mathrm{Fe}$, in the sense that the $\mathrm{Fe}$ abundance increases with metallicity, then we should expect the metal rich Bulge stars to show low $[\mathrm{O} / \mathrm{Fe}]$ ratios. 
Recently, McWilliam and Rich (1994) measured abundance ratios in metal rich stars in the bulge and, although these measures need to be confirmed, they showed that elements such $\mathrm{Mg}$ seem to be overabundant with respect to iron (i.e. $[\mathrm{Mg} / \mathrm{Fe}]>0$ ), thus indicating that the timescale for the collapse of the Bulge must have been shorter than in the solar neighbourhood.

If this is true it implies that the Galactic bulge is older than the solar vicinity region and it again points to the conclusion that the Milky Way should have formed from inside out. Another important aspect of the McWilliam and Rich results is that the $[\mathrm{Fe} / \mathrm{H}]$ abundances are all systematically lower by $0.25-0.3$ dex than the abundances given for the same stars by Rich (1988). One of the reasons for this could be that Rich (1988), who derived $[\mathrm{Fe} / \mathrm{H}]$ from the magnesium index $M_{g_{2}}$, had assumed $[\mathrm{Mg} / \mathrm{Fe}]=0.0$ in his stars. These lower $[\mathrm{Fe} / \mathrm{H}]$ indicate that the Galactic bulge is not as metal-rich as previously thought and that the average $[\mathrm{Fe} / \mathrm{H}]$ is roughly solar. Since the peak of Rich's (1988) distribution at +0.3 dex led Matteucci and Brocato (1990) to conclude that a flatter IMF than in the solar neighbourhood was required, this new result might mean that there is no necessity for claiming a different IMF in the bulge.

A very recent study by Minniti (1993) has shown that there is a correlation between kinematics and metallicity in Bulge stars, in the sense that the more metal-poor stars seem to have higher velocity dispersion and lower rotational velocity than the metal-rich population. On the basis of his results together with the existence of a Bulge metallicity gradient he concludes that the Bulge formed by dissipational collapse. As a consequence of this he argues that the Bulge should be younger than the halo. On the other hand, Renzini (1994) has argued that the existing data are consistent with the bulk of stars in the Bulge being at least as old as globular clusters stars.

\section{Conclusions}

It is still difficult to draw firm conclusions on the formation and evolution of our Galaxy. The dynamical and chemical evidence shown in the previous paragraphs give some idea of the complexity of the problem but they do not allow to distinguish between different hypotheses. Although we all agree that the formation of the Galactic disk must have been slow and dissipative, there is not a clear understanding about the timescale for the formation of the halo. Chemical arguments suggest that the halo collapse was fast, i.e. not longer than 1.5-2.0 Gyr, whereas dynamical and kinematical arguments alone do not allow one to understand this timescale. Estimates of the age of globular clusters together with the morphology of the Horizontal Branch 
suggest that the globular clusters of the outer halo have an age spread of 3-4 Gyr. Can this be taken as a probe of long collapse time for the whole halo? Perhaps there was a different mechanism of formation operating in the inner and the outer halo, in the sense that the inner halo might have formed on a short timescale of the order of the chemical one, thus confirming a picture similar to that proposed originally by ELS, whereas the outer one could have formed in a more chaotic way by accretion of external material as suggested by Searle and Zinn (1978), or just more slowly. In order to better understand these issues we would need more data on abundance ratios and metallicities especially for halo stars since the chemical method of dating the halo is less ambiguous than the kinematical information.

In conclusion, by considering all the kinematical and chemical information available on the halo, disk and bulge, it seems to us that the most likely picture for the formation of our Galaxy is the inside out model, where the Galaxy collapsed differentially, being faster in the innermost regions. This model can in fact explain the abundance gradients in the Galactic disk, the metallicity distribution and abundance ratios in the Bulge, the possible age spread in globular clusters as well as the observed spread in the abundances of disk stars.

\section{References}

Bessel, M.S., Sutherland, R.S., Ruan, K. (1991) Ap. J., 383, L7

Bolte, M. (1987) Ap. J., 319, 760

Buonanno, R., Corsi, C.E., Fusi Pecci, F., Fahlam, G.G., Richer, H. (1994), Ap. J. Lett., 430,121

Burkert, A., Truran, J.W., Hensler, G. (1992) Ap. J., 391, 651

Edmunds, M.G., Greenhow, R.M., Johnson, D., Kluckers V., Vila, M.B. (1991) M.N.R.A.S. 251, 33P

Edvardsson, B., Andersen, J., Gustafsson, B., Lambert, D.L., Nissen, P.E., Tomkin, J. (1993) A.A., 275, 101

Eggen, O.J., Lynden-Bell, D., Sandage, A.R. (1962) Ap. J., 136, 748

Ferrini, F., Matteucci, F., Pardi, C., Penco, U. (1992) Ap. J., 385, 138

François, P., Matteucci, F. (1993) A.A., 280, 136

Fuchs, B., Dettbarn, C., Wielen, R. (1994) Ergodic Concepts in Stellar Dynamics, eds. D. Pfenninger and V.G. Gurzadyan (Springer-Verlag)

Geisler, D., Friel, E.D. (1992) Astron. J., 104, 128

Gilmore, G., Reid, N. (1983) M.N.R.A.S., 202, 1025

Gilmore, G., Wyse, R.F.G. (1985) Astr. J., 90, 2015

Gilmore G., Wyse, R.F.G., Kuijken, K. (1989) Evolutionary Phenomena in Galaxies, eds. J.E. Beckman and B.E.J. Pagel, (Cambridge University Press), p.172

Grenon, M. (1987) J. Astrophys. Astr., 8, 123

Katz, N. (1992) Ap. J., 391, 502

Larson, R.B. (1969) M.N.R.A.S., 145, 405

Larson, R.B. (1976) M.N.R.A.S., 176, 31

Marquez, A., Schuster, W.J. (1994) preprint

Matteucci, F., Brocato, E. (1990) Ap. J., 365, 539

Matteucci, F., François, P. (1992) A. A., 262, L1

Matteucci, F., François, P. (1989) M.N.R.A.S., 239, 886 
Matteucci, F., Tornambè, A. (1985) A. A., 142, 13

Mc William, A., Rich, R.M. (1994) Ap.J. Suppl., 749

Minniti, D. (1993) Ph.D. Thesis, Steward Observatory, University of Arizona

Norris, J.E. (1986) Ap. J. Suppl., 61, 667

Pardi, C., Ferrini, F., Matteucci, F. (1994) Ap. J., in press

Renzini, A. (1994) A.A., 285, L5

Rich, R.M. (1988) Astron. J., 95, 828

Ryan, S.G., Norris, J.E. (1991) Astron. J., 101, 1835

Sandage, A. (1987) The Galaxy, eds. G. Gilmore and B. Carswell, (Reidel, Dordrecht), p.321

Sandage, A. (1990) J.R.A.S.C., 84, 70

Sandage, A., Fouts, G. (1987) Astron. J., 93, 74

Schuster, W.J., Nissen, P.E. (1989) A.A., 222, 69

Searle, L., Zinn, R. (1978) Ap. J., 225, 357

VandenBergh, D.A., Bolte, M., Stetson, P.B. (1990) Astron. J., 100, 445

Wielen, R. (1977) A.A., 60, 263

Wielen, R., Fuchs, B. (1985) IAU N. 106, The Milky Way Galaxy, eds. H. van Woerden et al., (Reidel, Dordrecht) p.481

Wielen, R., Dettbarn, C., Fuchs, B., Jahreiss, H., Radons, G. (1992) IAU Symp. 149, The Stellar Populations of Galaxies, p.81

Wilson, T.L., Matteucci, F. (1992) Astron. Astrophys. Rev., 4,1

Wyse, R.F.G., Gilmore, G. (1993) ASP Conf. Series N.48, The Globular Cluster-Galaxy Connection, eds. J.Brodie and G. Smith.

Yoshii, Y., Saio, H. (1979) Publ. Astron. Soc. Japan, 31, 339

Zinn, R. (1985) Ap. J., 293, 424

Zinn, R. (1993) ASP Conf. Series No. 48, The Globular Cluster-Galaxy Connection, eds. J.Brodie and G. Smith, p. 38

\section{DISCUSSION}

B. Gustaffson: I wish to clarify that we (Edvardsson et al. 1993) for a given stellar age group and galactocentric mean distance found an astonishingly small scatter in $[\alpha / \mathrm{Fe}]$, while the scatter in $[\mathrm{Fe} / \mathrm{H}]$ is significant, about 0.2 dex. This may be explicable as the result of orbital diffusion and a metallicity gradient in the disk, but that would then imply a gravitational potential field that is varying with time or has a considerable roughness (cf. the poster paper by B. Fuchs and R. Wielen for this symposium).

L. Ozernoy: The phenomenon of high velocity clouds is seemingly consistent with the continuing build-up of the disk. However, it is unclear yet what is the rate of this process. Could you quantify somehow what is the current infall rate onto the Galaxy?

Matteucci: Most of the galactic chemical evolution models are consistent with an average infall rate in the disk now of $\sim 1 \mathrm{M}_{\odot} / \mathrm{yr}$.

H. van Woerden: (about high-velocity clouds)[Remark made after Underhill and Gustaffson] For the role of high velocity clouds (HVCs) in the formation and evolution of the Milky Way disk, the following facts are rel- 
evant (cf. poster by Wakker et al. in this Symposium):

1. The mass inflow is of the order of a few solar masses per year.

2. The metallicities of individual HVCs are quite different, ranging at least from $10^{-3}$ to $10^{-1}$ times solar. This may include primordial origin, although for some HVCs no metal absorption lines have yet been found.

3. Distances are still unknown for most HVCs, although information is accumulating.

4. Both accretion of intergalactic material and circulation of gas, in a Galactic Fountain may play important roles in the diverse HVC phenomenon in addition to distance and metallicity information, we require models for the dynamics and evolution of HVCs (cf. Wakker 1990, thesis Groningen) 